\title{
Tyrosine phosphatases as a superfamily of tumor suppressors in colorectal cancer
}

\author{
Izabela Laczmanska ${ }^{\bowtie}$ and Maria M. Sasiadek \\ Genetics Department, Medical University, Wroclaw, Poland
}

\begin{abstract}
Phosphorylation and dephosphorylation processes catalyzed by numerous kinases and phosphorylases are essential for cell homeostasis and may lead to disturbances in a variety of vital cellular pathways, such as cell proliferation and differentiation, and thus to complex diseases including cancer. As over $80 \%$ of all oncogenes encode protein tyrosine kinases (PTKs), protein tyrosine phosphatases (PTPs), which can reverse the effects of tyrosine kinases, are very important tumor suppressors. Alterations in tyrosine kinase and phosphatase genes including point mutations, changes in epigenetic regulation, as well as chromosomal aberrations involving regions critical to these genes, are frequently observed in a variety of cancers. Colorectal cancer (CRC) is one of the most common cancers in humans. CRCs occur in a familial (about $15 \%$ of all cases), hereditary (about $5 \%$ ) and sporadic (almost 75-80\%) form. As genetic-environmental interrelations play an important role in the susceptibility to sporadic forms of CRCs, many studies are focused on genetic alterations in such tumors. Mutational analysis of the tyrosine phosphatome in CRCs has identified somatic mutations in PTPRG, PTPRT, PTPN3, PTPN13 and PTPN14. The majority of these mutations result in a loss of protein function. Also, alterations in the expression of these genes, such as decreased expression of PTPRR, PTPRO, PTPRG and PTPRD, mediated by epigenetic mechanisms have been observed in a variety of tumors. Since cancer is a social and global problem, there will be a growing number of studies on alterations in the candidate cancer genes, including protein kinases and phosphatases, to determine the origin, biology and potential pathways for targeted anticancer therapy.
\end{abstract}

Keywords: phosphatases, colorectal cancer, mutations, epigenetics

Received: 17 October, 2011; revised: 21 November, 2011; accepted: 05 December, 2011; available on-line: 06 December, 2011

\section{INTRODUCTION}

Cell homeostasis should be tightly controlled. One of the key mechanisms in this control is the process of phosphorylation and dephosphorylation catalyzed by numerous kinases and phosphatases. Phosphorylation/ dephosphorylation of a protein switches it between an active and inactive form, which is connected with conformational changes. The reversibility and rapidity of this process make it one of the main mechanisms in cell homeostasis (Arena et al., 2005; Tabernero et al., 2008).

Phosphorylation and dephosphorylation affect the formation of protein complexes by altering protein conformation and therefore are crucial in the regulation of receptors, ion channels, signaling proteins and transcriptional factors (Arena et al., 2005).

\section{TYROSINE KINASES AND PHOSPHATASES — GENERAL CHARACTERISTICS}

Kinases catalyze the transfer of a phosphate group from the coenzyme adenosine-5'-triphosphate (ATP) to specific molecules, e.g. proteins or lipids. Phosphorylases catalyze a reverse process, i.e. the removal of a phosphate group from a substrate (Arena et al., 2005; Tabernero et al., 2008). Phosphorylation is a process in which a negatively charged, hydrophilic phosphate $\left(\mathrm{PO}_{4}\right)$ group is usually added to a hydroxyl group of a serine, threonine or tyrosine residue in a protein through a phosphoester bond (O-phosphate), but can be also added to an aspartate, histidine and arginine residue or to some lipids. Both groups of enzymes are usually characterized by a conserved catalytic domain (Arena et al., 2005; Tabernero et al., 2008).

A non-enzymatic pathway of protein phosphorylation is also active in mammals. In this process, which plays a crucial role in the regulation of endocytosis, chemotaxis and apoptosis, pyrophosphorylation is mediated by inositol pyrophosphates (Saiardi et al., 2004).

Because of the huge number of kinases and phosphatases and their crucial role in cell homeostasis, the set of genes encoding kinases is named the kinome, while the one encoding phosphatases is called the phosphatome (Arena et al., 2005).

Tyrosine kinases and phosphatases are either receptor protein kinases/phosphatases or soluble non-receptor (intracellular) enzymes (Ârena et al., 2005). According to their catalytic mechanism PTPs belong to the following two groups: Cys-based (3 classes in humans, 103 genes) and Asp-based PTPs (4 genes in humans). Class I Cys-based PTPs comprise 99 enzymes, wherein classical PTPs are represented by 38 proteins i.e., 21 classical transmembrane PTPs and 17 non-receptor PTPs (NRPTPs), while dual specific phosphatases (DSPs) are represented by 61 enzymes (Alonso et al., 2004). Cytoplasmic NRPTPs consist of catalytic domains, special non-catalytic domains or domains that are involved in subcellular directing, ligand binding or activity regulation. Receptor-

e-mail: iza.laczmanska@gmail.com

Abbreviations: ATP, adenosine-5'-triphosphate; CAN genes, candidate cancer genes; CRC, colorectal cancer; DSP, dual-specific phosphatase; LMW, low molecular weight; PANTHER, Protein ANalysis THrough Evolutionary Relationships; PTK, protein tyrosine phosphatase; PTP, protein tyrosine kinase; NRPTP, non-receptor protein tyrosine phosphatase; RPTP, receptor protein tyrosine phosphatase; SCRC, sporadic colorectal cancer. 
like PTPs (RPTPs or PTPRs) are transmembrane proteins and are usually composed of an N-terminal extracellular domain, a single transmembrane domain and one or two highly conserved intracellular catalytic domains. PTPRs are employed in transmembrane signal transduction. Dual specific protein phosphatases (DSPs) catalyze the dephosphorylation of serine and threonine in addition to tyrosine residues (Alonso et al., 2004; Tabernero et al., 2008).

Class II Cys-based LMW-PTPs (low molecular weight; 1 gene in humans), whose expression has been observed in the majority of human tissues, are involved in controlling cytoskeleton, cell growth and adhesion. LMW-PTPs present a high structural, but not sequence, homology to class I PTPs (Alonso et al., 2004; Tabernero et al., 2008; Wang et al., 2004). Class III consists of 3 genes encoding cell cycle regulators (CDC25A, B and C) (Alonso et al., 2004).

As PTPs are involved in the regulation of so many biological pathways, there has been an increasing number of studies published recently on their role in normal and pathological processes (Alonso et al., 2004).

\section{PTP MUTATIONS AND COLORECTAL CANCER}

More than 300 genes have been identified up to now as being involved in carcinogenesis. The major impact cancer genes are oncogenes and tumor suppressor genes (Arena et al., 2005; Jacob et al., 2005; Julien et al., 2011). Approximately $80 \%$ of all oncogenes code for protein kinases (PTKs). Some tyrosine phosphatases (PTPs) may act as tumor suppressors reversing the negative effects of PTKs, while others may act as oncoproteins exerting a positive effect on signaling processes by dephosphorylation and activation of PTKs (Jacob et al., 2005; Julien et al., 2011).

Colorectal cancer (CRC) is one of the most common human cancers, affecting both women and men. Colorectal cancers occur in a sporadic, familial and hereditary form (Cheah 2009; Wicki et al., 2010). Differences in the molecular pathways underlying the development of various CRC subgroups led to the hypothesis that they constituted different disease entities (Samowitz 2008).

The majority of CRCs are sporadic (about 80\%), but studies on monozygotic twins showed that both genetic and environmental factors modulate individual susceptibility to CRC (Cheah, 2009). Thus, an individual's susceptibility to sporadic CRC (SCRC) depends on the combination of environmental exposure to potentially carcinogenic agents (e.g. diet, smoking) and low penetrant genes that modulate the response to these environmental factors (Toland et al., 2008).

The most frequent genetic alterations in SCRC are $A P C$ somatic mutations detected in up to $80 \%$ of sporadic cases. Loss of APC function results in an accumulation of nuclear beta-catenin and thus in the activation of the Wnt signaling pathway (Samowitz 2008; Cheah 2009; Wicki et al., 2010). Recent studies on anti-EGFR therapy in patients with metastatic CRCs revealed that mutations in genes such as $B R A F, K R A S$ and PIK3C result in resistance to this therapy (Lurkin et al., 2010).

Genome-wide studies employing microarray technology have revealed a variety of candidate genes suspected of being important in the development, progression and metastasis of SCRC (Wang et al., 2004; Toland et al., 2008; Mokarram et al., 2009; Kim et al., 2011; van Roon et al., 2011). Many of them code for kinases and phos- phatases. A variety of genetic, as well as epigenetic, alterations affecting their function and/or expression have been identified recently (Wang et al., 2004; Arena et al., 2005; Jacob et al., 2005; Toland et al., 2008).

Mutational analysis of the tyrosine phosphatome in CRC performed in an elegant experiment by Wang et al. (2004) allowed them to identify 83 somatic mutations in the following six genes: three members of the RPTP subfamily (PTPRF, PTPRG, PTPRT) and three members of the NRPTPs (non-receptor) subfamily (PTPN3, PTPN13, PTPN14) in $26 \%$ of the examined CRCs. Moreover, the authors identified six other PTP mutations in lung, gastric and breast cancer cells. The majority of the mutations detected were related to a loss of enzyme function (Wang et al., 2004).

Studies on the function of PTPRT, which was reported to be most frequently mutated among the analyzed phosphatases in CRC (Wang et al., 2004), have showed that paxillin is a direct substrate of PTPRT (Zhao et al., 2010). Phosphorylation of paxillin was observed at multiple sites including 8 different tyrosine residues. The oncogenic function of phospho-paxillin is exerted by deregulation of cell adhesion, migration, proliferation and apoptosis (Zhao et al., 2010). However, PTPRT also acts as a tumor suppressor by inactivating STAT3 (signal transducer and activator of transcription 3), since dephosphorylation of STAT3 Y705 residue results in a decrease in its activity (Zhang et al., 2007).

Tumor suppressor effect in colon cancer has also been observed for the following protein tyrosine phosphatases: DEP-1 (density enhanced phosphatase), which inhibits proliferation and migration of cancer cells and PTPRD which is involved in cell adhesion and suppression of cell migration (Balavenkatraman et al., 2006; Funato et al., 2011).

Frameshift mutations in six phosphatases (PTPN21, PTPN23, PTPN5, PTPRA, PTPRE and PTPRS) in colorectal cancers were also reported by Korff et al. (2008). However, these authors did not observe any impact of PTP mutations on CRC tumorigenesis. The difference between the results of Korff et al. (2008) and Wang et al. (2004) may result from different molecular characteristics of the tumors studied, as Korff et al. (2008) analyzed CRCs showing microsatellite instability (MSI), while Wang et al. (2004) did not specially select such CRCs (Wang et al., 2004; Korff et al., 2008; Julien et al., 2011). In in vivo studies, the gene coding for PTPRJ has been identified as a gene associated with susceptibility to colon cancer (Ruivenkamp et al., 2003). Moreover, frequent LOH (loss of heterozygosity) at position $11 \mathrm{p} 11$ (the PTPRJ locus) has been frequently observed in SCRCs, suggesting involvement of this gene in the carcinogenesis of CRCs (Ruivenkamp et al., 2003; Puijenbroek et al., 2005).

Studies on the role of dual specific phosphatase (DPTP) genes in gastric and colorectal cancer have been performed on tumors with and without MSI. Deletions and duplications of one nucleotide (frameshift mutations) were detected in the nucleotide repeats in CDC14A and MTMR3, but only in the MSI-H cancer group. This may suggest that alterations in these two genes may contribute to the oncogenesis of colon and gastric cancer with MSI (Song et al., 2010).

\section{EPIGENETIC REGULATION OF PTP ACTIVITY IN CRCS}

Alterations in DNA methylation leading to chromatin modification and thus to changes in gene expression be- 
long to the most important molecular mechanisms in the development and progression of tumors (Motiwala et al., 2003; Kim et al., 2011).

Hypomethylation leading to the activation of protooncogenes, as well as hypermethylation resulting in the inactivation of tumor suppressors, have been frequently observed in cancers (Motiwala et al., 2003; Menigatti et al., 2009; Kim et al., 2011). Changes in PTP expression have been frequently observed among other genetic alterations in CRCs. PTPRR inactivation, leading to a reduction in the mRNA level has been observed in precancerous colorectal lesions, colorectal tumors, colorectal cell lines and also in liver metastases. PTPRR encodes the transmembrane protein tyrosine phosphatase, a receptor-type phosphatase, which is employed in the RAS/ RAF/MAPK/ERK pathway (Menigatti et al., 2009).

Hypermethylation, and thus a decrease in the expression of PTPs, has been observed in a variety of tumors, such as hepatocellular rat carcinomas (PTPRO), cutaneous T-cell lymphomas (PTPRG) and glioblastomas (PTPRD) (Motiwala et al., 2003; Wang et al., 2004; van Doorn et al., 2005; Veeriah et al., 2009). PTPRG and PTPRO, which play a role in apoptosis, terminal differentiation and cell cycle, are candidates for tumor suppressor genes in colon, renal and lung carcinomas (Motiwala et al., 2003; Wang et al., 2004; van Doorn et al., 2005). PTPRD dephosphorylates the oncoprotein STAT3 and thus regulates the STAT3 pathway. Because of its proximity to CDKN2A on chromosome 9p, the loss of PTPRD was thought to be associated with the loss of the CDKN2A gene. Subsequent analysis proved that homozygous deletion of PTPRD without the deletion of neighboring genes is frequently observed in a variety of tumors (Veeriah et al., 2009).

Studies of candidate cancer genes (CAN genes) in CRCs revealed promoter hypermethylation in over $65 \%$ of those genes (Mokarram et al., 2009). It has been proved that a loss of PTPRD activity can be caused by classical mechanisms, which are critical for tumor suppressor genes (Veeriah et al., 2009).

Array-based technology is one of the most powerful tools in cancer research enabling a comprehensive analysis of a huge number of biological factors in a short time.

Microarray studies of methylation in colorectal cancer analyzing $27578 \mathrm{CpG}$ sites in 14000 genes, revealed hypermethylation of $621 \mathrm{CpG}$ sites located in the promoter regions of a variety of genes (Kim et al., 2011). Downregulation of the expression of hypermethylated genes has been observed. However, these results were not statistically significant. Functional analysis of these genes using the PANTHER (Protein ANalysis THrough Evolutionary Relationships) classification system revealed their involvement in signal transduction, mRNA transcription, cell-cell communication and surface-receptor mediated signal transduction, as well as cell adhesion, proliferation and differentiation (Kim et al., 2011). Kinases and phosphorylases are involved in most of these processes (Arena et al., 2005).

Aberrant methylation of the $\mathrm{CpG}$-rich region in intron 1 of PTPRG has been detected in colon adenomas developing into carcinomas in both hereditary nonpolyposis colon cancer and in sporadic colorectal tumors. Despite the fact that this alteration has not been connected with deregulation of PTPRG expression, the high specificity $(96 \%)$ and sensitivity $(94 \%)$ of the status of PTPRG methylation open the possibility of its applica- tion as a diagnostic marker in the early detection of CRC (van Roon et al., 2011).

\section{CONCLUSION}

The important role of phosphatases and kinases as both oncogenes and tumor suppressors, as well as the apparent reversibility of their oncogenic effects in phosohorylation/defosphorylation processes make these genes/proteins interesting potential targets in anticancer therapy. Therefore, an increasing number of studies on their role in cancerogenesis have been published recently.

\section{Acknowledgements}

This paper has been supported by the State Committee for Scientific Research, Polish Ministry for Scientific Research and Information Technology (grant no. N N401 601438), 2010-2013 and by a research fellowship within the "Development program of Wroclaw Medical University" funded by the European Social Fund, Human Capital, National Cohesion Strategy" (contract no. UDA-POKL.04.01.01-00-010/08-00)".

The authors are grateful to David Ramsey, University of Limerick, for final editing of the manuscript.

\section{REFERENCES}

Alonso A, Sasin J, Bottini N, Friedberg I, Friedberg I, Osterman A, Godzik A, Hunter T, Dixon J, Mustelin T (2004) Protein tyrosine phosphatases in the human genome. Cell 117: 699-711.

Arena S, Benvenuti S, Bardelli (2005) Genetic analysis of the kinome and phosphatome in cancer. Cell Mol Life Sci 62: 2092-2099.

Balavenkatraman KK, Jandt E, Friedrich K, Kautenburger T, PoolZobel BL, Ostman A, Böhmer FD (2006) DEP-1 protein tyrosine phosphatase inhibits proliferation and migration of colon carcinoma cells and is upregulated by protective nutrients. Oncogene 12: 63196324.

Cheah PY (2009) Recent advances in colorectal cancer genetics and diagnostics. Crit Rev Oncol Hematol 69: 45-55.

van Doorn R, Zoutman WH, Dijkman R, de Menezes RX, Commandeur S, Mulder AA, van der Velden PA, Vermeer MH, Willemze R, Yan PS, Huang TH, Tensen CP (2005) Epigenetic profiling of cutaneous T-cell lymphoma: promoter hypermethylation of multiple tumor suppressor genes including BCL7a, PTPRG, and p73. J Clin Oncol 23: 3886-3896.

Funato K, Yamazumi Y, Oda T, Akiyama T (2011) Tyrosine phosphatase PTPRD suppresses colon cancer cell migration in coordination with CD44. Exp Ther Med 2: 457-463.

Jacob ST, Motiwala T (2005) Epigenetic regulation of protein tyrosine phosphatases: potential molecular targets for cancer therapy. Cancer Gene Ther 12: 665-672.

Julien SG, Dubé N, Hardy S, Tremblay ML (2011) Inside the human cancer tyrosine phosphatome. Nat Rev Cancer 11: 35-49.

Kim YH, Lee HC, Kim SY, Yeom YI, Ryu KJ, Min BH, Kim DH, Son HJ, Rhee PL, Kim JJ, Rhee JC, Kim HC, Chun HK, Grady WM, Kim YS (2011) Epigenomic analysis of aberrantly methylated genes in colorectal cancer identifies genes commonly affected by epigenetic alterations. Ann Surg Oncol 18: 2338-2347.

Korff S, Woerner SM, Yuan YP, Bork P, von Knebel Doeberitz M, Gebert J (2008) Frameshift mutations in coding repeats of protein tyrosine phosphatase genes in colorectal tumors with microsatellite instability. BMC Cancer 8: 329.

Lurkin I, Stoehr R, Hurst CD, van Tilborg AA, Knowles MA, Hartmann A, Zwarthoff EC (2010) Two multiplex assays that simultaneously identify 22 possible mutation sites in the KRAS, BRAF, NRAS and PIK3CA genes. PLoS One 5: e8802.

Menigatti M, Cattaneo E, Sabates-Bellver J, Ilinsky VV, Went P, Buffoli F, Marquez VE, Jiricny J, Marra G (2009) The protein tyrosine phosphatase receptor type $\mathrm{R}$ gene is an early and frequent target of silencing in human colorectal tumorigenesis. Mol Cancer 8: 124.

Mokarram P, Kumar K, Brim H, Naghibalhossaini F, Saberi-Firoozi M, Nouraie M, Green R, Lee E, Smoot DT, Ashktorab H (2009) Distinct high-profile methylated genes in colorectal cancer. PLOS One 4: e7012.

Motiwala T, Ghoshal K, Das A, Majumder S, Weichenhan D, Wu YZ, Holman K, James SJ, Jacob ST, Plass C (2003) Suppression of the 
protein tyrosine phosphatase receptor type $\mathrm{O}$ gene (PTPRO) by methylation in hepatocellular carcinomas. Oncogene 22: 6319-6331.

van Puijenbroek M, Dierssen JW, Stanssens P, van Eijk R, CletonJansen AM, van Wezel T, Morreau H (2005) Mass spectrometrybased loss of heterozygosity analysis of single-nucleotide polymorphism loci in paraffin embedded tumors using the MassEXTEND assay: single-nucleotide polymorphism loss of heterozygosity analysis of the protein tyrosine phosphatase receptor type $J$ in familial colorectal cancer. J Mol Diagn 7: 623-630.

van Roon EH, de Miranda NF, van Nieuwenhuizen MP, de Meijer EJ, van Puijenbroek M, Yan PS, Huang TH, van Wezel T, Morreau H, Boer JM (2011) Tumour-specific methylation of PTPRG intron 1 locus in sporadic and Lynch syndrome colorectal cancer. Eur J Hum Genet 19: 307-312.

Ruivenkamp C, Hermsen M, Postma C, Klous A, Baak J, Meijer G, Demant P (2003) LOH of PTPRJ occurs early in colorectal cancer and is associated with chromosomal loss of 18q12-21. Oncogene 22: 3472-3474.

Saiardi A, Bhandari R, Resnick AC, Snowman AM, Snyder SH (2004) Phosphorylation of proteins by inositol pyrophosphates. Science 306: 2101-2105.

Samowitz WS (2008) Genetic and epigenetic changes in colon cancer. Exp Mol Pathol 85: 64-67.

Song SY, Kang MR, Yoo NJ, Lee SH (2010) Mutational analysis of mononucleotide repeats in dual specificity tyrosine phosphatase genes in gastric and colon carcinomas with microsatellite instability. APMIS 118: 389-393.

Tabernero L, Aricescu AR, Jones EY, Szedlacsek SE (2008) Protein tyrosine phosphatases: structure-function relationships. FEBS J 275: 867-882.
Toland AE, Rozek LS, Presswala S, Rennert G, Gruber SB (2008) PTPRJ haplotypes and colorectal cancer risk. Cancer Epidemiol Biomarkers Prev 17: 2782-2785.

Veeriah S, Brennan C, Meng S, Singh B, Fagin JA, Solit DB, Paty PB, Rohle D, Vivanco I, Chmielecki J, Pao W, Ladanyi M, Gerald WL, Liau L, Cloughesy TC, Mischel PS, Sander C, Taylor B, Schultz N, Major J, Heguy A, Fang F, Mellinghoff IK, Chan TA (2009) The tyrosine phosphatase PTPRD is a tumor suppressor that is frequently inactivated and mutated in glioblastoma and other human cancers. Proc Natl Acad Sci USA 106: 9435-9440.

Wang Z, Shen D, Parsons DW, Bardelli A, Sager J, Szabo S, Ptak J, Silliman N, Peters BA, van der Heijden MS, Parmigiani G, Yan H, Wang TL, Riggins G, Powell SM, Willson JK, Markowitz S, Kinzler KW, Vogelstein B, Velculescu VE (2004) Mutational analysis of the tyrosine phosphatome in colorectal cancers. Science 304: 1164-1166.

Wicki A, Herrmann R, Christofori G (2010) Kras in metastatic colorectal cancer. Swiss Med Wkly 140: 13112.

Zhang X, Guo A, Yu J, Possemato A, Chen Y, Zheng W, Polakiewicz RD, Kinzler KW, Vogelstein B, Velculescu VE, Wang ZJ (2007) Identification of STAT3 as a substrate of receptor protein tyrosine phosphatase T. Proc Natl Acad Sci USA 104: 4060-4064.

Zhao Y, Zhang X, Guda K, Lawrence E, Sun Q, Watanabe T, Iwakura Y, Asano M, Wei L, Yang Z, Zheng W, Dawson D, Willis J, Markowitz SD, Satake M, Wang Z (2010) Identification and functional characterization of paxillin as a target of protein tyrosine phosphatase receptor T. Proc Natl Acad Sci USA 107: 2592-2597. 